\title{
A Throughput-Aimed MAC Protocol with QoS Provision for Cognitive Ad Hoc Networks
}

\author{
Yichen WANG ${ }^{\dagger}$, Nonmember, Pinyi REN ${ }^{\dagger a}$, and Guangen $\mathrm{WU}^{\dagger}$, Members
}

SUMMARY In this letter, we propose a Throughput-aimed MAC Protocol with Quality of Service (QoS) provision (T-MAC) for cognitive Ad Hoc networks. This protocol operates based on the Time Division Multiple Access (TDMA) slot assignments and the power control mechanism, which can improve the QoS provision and network throughput. Our simulation results show that the T-MAC protocol can efficiently increase the network throughput and reduce the access delay.

key words: cognitive radio, Ad Hoc networks, Medium Access Control (MAC), Quality of Service (QoS)

\section{Introduction}

With the rapid development of wireless communication technologies, it is promising yet challenging to apply $\mathrm{Cog}$ nitive Radio (CR) technologies to improve the system capacity and performance of Ad Hoc networks. In the existing cognitive MAC protocols [1]-[4], several major issues still remain as open problems. First, the ACK and Data packets usually cannot be protected simultaneously. Second, the Multi-Channel Hidden Terminal Problem (MCHTP) has not been well solved. Third, the Multi-Channel Exposed Terminal Problem (MCETP), despite its vital importance, has been neither well understood nor comprehensively studied.

To overcome these problems, we propose a MAC protocol, named Throughput-aimed MAC Protocol with QoS provision (T-MAC). The proposed protocol has three main features. First, it defines synchronized super frames which reduce the control packet collision probability. Second, the space reuse efficiency is increased and concurrent transmissions are supported by the power control mechanism. Third, special acknowledgement frames, which are responsible for protecting ACK packets, are considered. Moreover, we prove that the T-MAC protocol can efficiently solve both the MCHTP and MCETP, as well as alleviate the effects brought by the MCHTP solving process.

The rest of this letter is organized as follows. Section 2 introduces the system model. Section 3 proposes the T-MAC protocol. Section 4 analyzes the proposed protocol and presents simulation results. The paper concludes with Sect. 5.

\footnotetext{
Manuscript received October 23, 2009.

Manuscript revised January 30, 2010.

†The authors are with the Xi' an Jiaotong University, China.

a)E-mail: pyren@mail.xjtu.edu.cn

DOI: 10.1587/transcom.E93.B.1426
}

\section{System Model}

Our system contains two parts, namely Primary Users (PUs) and cognitive Ad Hoc networks (CAHNs). PUs are authorized to use licensed spectrum for communication. The objective of CAHNs is to opportunistically utilize some of those licensed spectrum for packet transmission without interrupting PUs' communication. In order to prevent PUs from suffering potential interference, at any time, we only allow CAHNs to utilize those licensed spectrum that PUs do not use.

In this letter, we consider a CAHN with Node_Num $\mathrm{CR}$ nodes. The CAHN has one Common Control Channel (CCC) with central frequency $f_{0}$ for exchanging control packets. The licensed spectrum is divided into Ch_Num orthogonal frequency data channels with central frequencies $\left\{f_{1}, \cdots, f_{\text {Ch_Num }}\right\}$. PUs use data channels as ON/OFF model [1], [4], which means PUs composes a Time Division Multiple Address (TDMA) system. In this model, data channels are divided into synchronized time slots called channel slots. The spectrum usage statistics of each PU remains unchanged in each channel slot. Therefore, CR nodes need to sense data channels at the beginning of each channel slot in order to decide which channel they can use in the current channel slot. low:

The assumptions for CR Nodes are summarized as be-

(1) Each Node is equipped with a single half-duplex radio that can realize sensing, transmitting and receiving, but with only one function at any time.

(2) Nodes send control packets with maximal power $P_{\max -s}$, and transmit Data and ACK packets with adjusted power.

(3) If the power of a packet reaches to one node is less than $P_{\min }^{\text {infer }}$, then the packet will not introduce any interference to that node.

(4) One node can successfully receive a packet only if the Signal-to-Interference Ratio (SIR) of that packet is larger than $S I R_{t h}$.

(5) Suppose the link between two nodes is symmetrical and the link gain remains unchanged in any channel slot.

\section{T-MAC Protocol}

Figure 1 shows the super frame of the proposed protocol. 


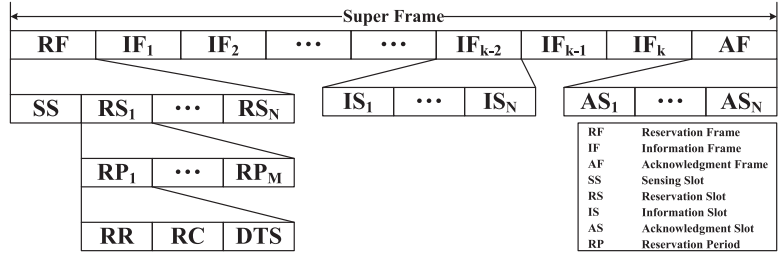

Fig. 1 Super frame of T-MAC protocol.

Each super frame consists of one Reservation Frame, $K$ Information Frames and one Acknowledgment Frame. The super frames are synchronized and has the same duration with channel slots. Each reservation frame contains one Sensing Slot and $N$ Reservation Slots. Correspondingly, each Information Frame and Acknowledgment Frame consists of $N$ Information Slots and Acknowledgment Slots. In order to decide which channel is available in current channel slot, all nodes must sense data channels in Sensing Slots. Each Reservation Slot is dedicated to the reservation of the corresponding information slot and acknowledgment slot. Each reservation period is a reservation process implemented through three-phase dialogues, named Reservation Request, Reservation Confirmation and Deciding To Send. Setting several reservation periods in one Reservation Slot is to enhance the reservation success rate, which can facilitate concurrent transmissions.

The proposed protocol is a slotted protocol, so strict synchronization is needed not only to guarantee the synchronization among nodes in the CAHN but also to make the CAHN to synchronize with PUs. In practice, we could use GPS to guarantee accurate synchronization. Although it will bring some extra cost, the network performance will significantly improved.

Each CR node maintains two lists named Channel Usage List of Data Packets (CUL-D) and Channel Usage List of ACK Packets (CUL-A). The objective is to make each node keep track of the channel usage condition of PUs and neighbor nodes. The two lists have the same structure and record a item for each data channel. Each item contains "Channel Number," "Maximal Allowed Transmitting Power," "Minimal Available Receiving Power," and "Channel State." If one data channel is occupied by PUs, then the corresponding "Channel State" is set by "1." Those channels that are marked by " 1 " can not be used by the CAHN. The two lists are updated according to the sensing results and the correlative information of neighbor nodes.

As mentioned above, each successful reservation process contains three-phase dialogues. The details of each phase are given below. Suppose $i$ is the source, and $j$ is the destination.

1. Reservation Request Phase. In this phase, node $i$ firstly inspects its CUL-D and CUL-A to affirm whether both lists contain available data channels. If such channels exist, then a Reservation Request (RR) packet, which contains node $i$ ' CUL-D and CUL-A, is sent to node $j$.

2. Reservation Confirmation Phase. If node $j$ received the RR packet, then it compares its CUL-D with node $i$ 's to find common available data channels for transmitting the Data packet. The same procedure is also done for the two CUL-As to determine common available data channels for the ACK packet transmission. The sets composed by all common available data channels in CUL-Ds and CUL-As are noted by $\Omega_{D}^{i j}$ and $\Omega_{A}^{j i}$. If the both sets are not null sets, the link gain $h_{i j}^{0}$ between $i$ and $j$ in CCC can be obtained through (1).

$$
h_{i j}^{0}=P_{r}^{R R} / P_{\max -s}
$$

where $P_{r}^{R R}$ and $P_{\max -s}$ are the receiving and transmitting power of the RR packet. Suppose the propagation model between two nodes is the two-ray model. Then, for each channel $k$ in $\Omega_{D}^{i j}$, the link gain $h_{i j}^{k}$ between node $i$ and $j$ in the $k$ th data channel as well as the corresponding transmitting power $P_{i j}^{k}$ of node $i$ can be acquired by (2) and (3).

$$
\begin{aligned}
& h_{i j}^{k}=h_{i j}^{0} \times\left(f_{0} / f_{k}\right)^{2} \\
& P_{i j}^{k}=P_{\text {min }-r}^{j, k} / h_{i j}^{k}
\end{aligned}
$$

where $f_{0}$ and $f_{k}$ are the frequencies of CCC and data channel $k, P_{\min -r}^{j, k}$ is the minimum receiving power that can guarantee the successful reception for node $j$. Finally, node $j$ can select the data channel $D C_{D(i j)}$ for transmitting the Data packet as (4).

$$
D C_{D(i j)}=\operatorname{argmin}\left\{D C_{k} \mid P_{i j}^{k} \leq P_{\max -s}^{i, k}, k \in \Omega_{D}^{i j}\right\}
$$

where $P_{\max -s}^{i, k}$ is the maximal transmitting power allowed to use in data channel $k$ for node $i$. The channel $D C_{A(j i)}$ for transmitting the ACK packet and the transmitting power can be decided from $\Omega_{A}^{j i}$ in the same way.

After completing above operations, a Reservation Confirmation (RC) packet, which contains the information about the selected channels and corresponding power, is sent by node $j$.

3. Deciding To Send Phase. While receiving the RC packet, node $i$ sends a Deciding To Send (DTS) packet, which contains the same information with the RC packet.

In T-MAC protocol, neighbor nodes overheard RC or DTS packets should also update correlative information contained in their CUL-As and CUL-Ds.

1. Overhearing RC Packets. Suppose node $k$ overhears the RC packet sent by node $j$. If node $k$ has already made a successful reservation for the current reservation slot, do nothing. Otherwise, node $k$ uses (5) to calculate the maximal allowed transmitting power $P_{\max -s}^{k, D(i)}$ in $D C_{D(i j)}$, and (6) to update the minimal available receiving power $P_{\min -r}^{k, A(j i)}$ in $D C_{A(j i)}$.

$$
\begin{aligned}
& P_{\max -s}^{k, D(i j)}=P_{\min }^{i n f e r} / h_{j k}^{D(i j)} \\
& P_{\min -r}^{k, A(j i)}=S I R_{t h} \cdot\left(\frac{P_{\min -r}^{k, A(j i)}}{S I R_{t h}}+h_{j k}^{A(j i)} \cdot P_{j i}^{A(j i)}\right)
\end{aligned}
$$

where $P_{j i}^{A(j i)}$ is the transmitting power of the ACK packet for 
node $j$.

2. Overhearing DTS Packets. Suppose node $m$ overhears the DTS packet sent by node $i$. If a successful reservation for the reservation slot had already been made by node $m$, do nothing. Otherwise, node $m$ adjusts the minimal available receiving power in $D C_{D(i j)}$ and the maximal allowed transmitting power in $D C_{A(j i)}$ through the same method.

\section{Protocol Analysis and Simulation Results}

\subsection{Protocol Analysis}

The Multi-Channel Hidden Terminal Problem (MCHTP) is an important issue in CAHNs. The MCHTP is that, a sending node may not hear the ongoing reception of its neighbor receiving node because different nodes can operate on different channels, so the sending node may transmit its Data packet on the channel that is the same with its neighbor receiving node. Therefore Data packet collision will be caused. It is clear that the MCHTP is harmful for the network. The MCHTP can be solved through control packets exchange, but all the neighbor nodes must defer their data transmission when a node is receiving its packets. Therefore, although Data packet collision can be avoided, the network throughput will also be reduced.

While applying the T-MAC protocol, since any node can get the reservation information of its neighbor nodes, the MCHTP will not occur. Moreover, any node has the opportunity to use those data channels that are identical with its neighbor nodes due to the power control mechanism. Therefore, the effects brought by the MCHTP solving process can be efficiently reduced.

The Multi-Channel Exposed Terminal Problem (MCETP) is the other critical issue, but has been neither well understood nor comprehensively studied. So-called MCETP is that, when one sending node discovers its neighbor nodes are sending Data packets in some data channels through carrier sense, the sending node will give up choosing those channels for transmission. Obviously, the available channels for the sending node are remarkably reduced. Therefore, the network throughput will be seriously harmed.

In T-MAC protocol, with strict TDMA slot division and synchronizing operation, all nodes are guaranteed in the same state at any time. Therefore, two neighbor sending nodes can make the reservation for the same reservation slot during the same reservation period and their available channel resources will not change. Therefore, the T-MAC protocol can thoroughly figure out the MCETP.

\subsection{Simulation Results}

In this section, we will present the performance of T-MAC and DSA-MAC. The latter one is the combination of IEEE 802.11 DCF which is the most typical protocol for Ad Hoc networks and sensing function. In DSA-MAC, node pairs compete for data channels through exchanging three control

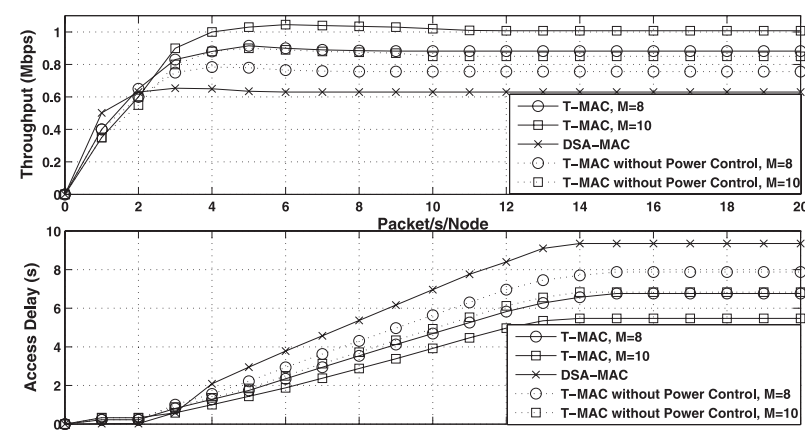

Fig. 2 Performance comparison

packets, which are RTS, CTS, and RES respectively. Moreover, we will give the performance of T-MAC without power control mechanism for evaluating the effect of power control mechanism.

In our simulation, 49 nodes are uniformly distributed in an area of $700 \times 700 \mathrm{~m}^{2}$. Each Data packet contains 1280 Bytes. $K$ and $N$ are set as 10 and 16. Each information slot is $1.024 \mathrm{~ms}$. The RR packet is 300 bits, and the RC, DTS, and ACK packets are all 250 bits. There are 3 data channels and $1 \mathrm{CCC}$ in the network. The data rate $R$ of these channels are all $1 \mathrm{Mbps}$. At the beginning of each super frame, PUs rechoose channels to use. For each channel, it is occupied by PUs with probability $P$. The larger $P$ is, the more frequent PUs' activity is. Here we set $P=0.5$, which means PUs' activity is moderate and half of channels can be used for the CAHN statistically.

Figure 2 shows the aggregated network throughput and access delay, where $M$ is the number of reservation periods contained in one reservation slot. From the result we can see, no matter whether introduce the power control mechanism, the performance of T-MAC is better than DSA-MAC except the initial phase. When we do not consider the power control mechanism, the advantage of T-MAC comes from three aspects. First, the control packet collisions can be reduced through distributing the control packet sending into several reservation slots. Second, the T-MAC can use two different channels for transmitting Data and ACK packets, which can improve the reservation success rate. Third, the MCHTP and MCETP can be solved.

While applying the power control mechanism, we can find that the performance of T-MAC is further improved. The reason is, power control can reduce the mutual interference among neighbor nodes and increase the available channels of each node. Therefore, more concurrent transmissions can happen simultaneously. In another word, the negative effect brought by the MCHTP solving process is considerable alleviated.

Because all nodes are able to successfully send their data packets in time no matter which protocol is applied when the network traffic is low, but the data packets can be sent only after one reservation frame in the T-MAC, the network performance of T-MAC is worse than the DSA-MAC in the initial phase. 


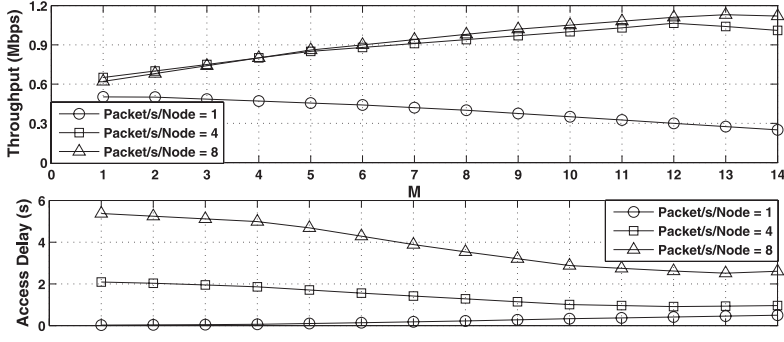

Fig. 3 Performance of $M$ versus throughput and access delay.

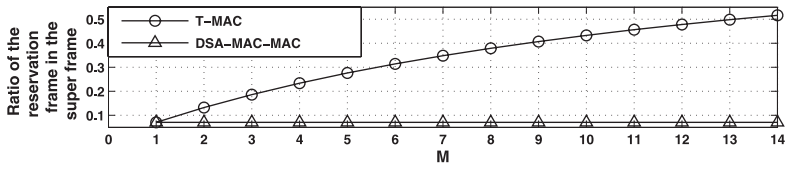

Fig. 4 Ratio of the reservation frame in the super frame.

Figure 3 shows the performance of $M$ versus throughput and access delay. We observe that better performance of T-MAC can be obtained with setting more reservation periods in one reservation slot when the traffic is high. The reason is that larger probability of reserving the same reservation slot for neighbor nodes and more parallel transmissions can be realized with larger $M$. However, with the continuous increasing of $M$, the performance become worse. This is because resources in the network are limit, even if the opportunities for concurrent transmissions become larger.

Finally, let's analyze the redundancy of reservation. In T-MAC, the duration of the reservation frame and super frame are given in (7) and (8).

$$
\begin{aligned}
& T_{R F}=M \cdot N \cdot\left(L_{R R}+L_{R C}+L_{D T S}\right) / R \\
& T_{\text {super }}=T_{R F}+M \cdot N \cdot T_{I S}+N \cdot L_{A C K} / R
\end{aligned}
$$

where $L_{R R}, L_{R C}, L_{D T S}$, and $L_{A C K}$ are the length of the RR, RC, DTS, and ACK packet.

Therefore, the ratio of the reservation frame in the super frame can be acquired through (9).

$$
r_{1}=T_{R F} / T_{\text {super }}=12.8 M /(12.8 M+167.84)
$$

In DSA-MAC, the ratio of the reservation time and the time of one successful transmission is given in (10).

$$
r_{2}=\frac{T_{R T S}+T_{C T S}+T_{R E S}}{T_{R T S}+T_{C T S}+T_{R E S}+T_{D A T A}+T_{A C K}}
$$

The ratio derived from (9) and (10) are depicted in
Fig. 4. From this result, we find that the ratio of T-MAC increases with $M$ while the DSA-MAC remaining the same. Although the reservation time for one transmission of $\mathrm{T}$ MAC is lager, the redundant time can be used for concurrent transmission, whereas the source and destination will defer the transmission of their neighbor nodes in DSA-MAC. Therefore, the performance of T-MAC is significantly improved.

\section{Conclusions}

A MAC protocol for cognitive Ad Hoc networks, named T-MAC, is proposed in this letter. The proposed protocol has four characteristics. First, the control packet collisions among neighbor nodes are reduced through strict time slot management. Second, the Data and ACK packets are protected simultaneously through special acknowledgment frames. Third, the space reuse efficiency are improved by the power control mechanism. Fourth, the MCETP and MCHTP can be solved, and the negative effect brought by the MCHTP solving process is alleviated. Finally, we verify that the T-MAC can provide better system capacity and QoS provision through simulation.

\section{Acknowledgments}

The paper is supported by National Natural Science Foundation of China (Grant No. 60832007), National Hi-Tech Research and Development Plan of China (Grant No. 2009AA011801), and National Key Laboratory Foundation (Grant No. 9140C5303030803).

\section{References}

[1] J. Jia, Q. Zhang, and X. Shen, "HC-MAC: A hardware-constrained cognitive MAC for efficient spectrum management," IEEE J. Sel. Areas Commun., vol.26, no.1, pp.95-105, Jan. 2008.

[2] C. Cordeiro and K. Challapali, "C-MAC: A cognitive MAC protocol for multichannel wireless networks," IEEE DySPAN, pp.147-157, Dublin, Ireland, 2007.

[3] Y.R. Kondareddy and P. Agrawal, "Synchronized MAC protocol for multihop cognitive radio networks," IEEE ICC, pp.3198-3202, Beijing, China, 2008.

[4] H. Su and X. Zhang, "Cross-layer based opportunistic MAC protocols for QoS provisionings over cognitive radio wireless networks," IEEE J. Sel. Areas Commun., vol.26, no.1, pp.118-129, Jan. 2008.

[5] C.X. Zhu and M.S. Corson, "A five-phase reservation protocol (FPRP) for mobile ad hoc networks," Wirel. Netw., vol.7, no.4, pp.371-384, July 2001. 